\title{
The role of allergoids in allergen immunotherapy: from injective to sublingual route
}

E. Compalati ${ }^{1}$, C. Incorvaia ${ }^{2}$, C. Cavaliere $^{3}$, S. Masieri ${ }^{4}$, A. Gargiulo ${ }^{5}$, G. Mistrello ${ }^{6}$, F. Frati $^{1}$

${ }^{1}$ Scientific and Medical DPT Lofarma Milano, Milan, Italy

${ }^{2}$ Cardiac/Pulmonary Rehabilitation, ASST Pini/CTO, Milan, Italy.

${ }^{3}$ Department of Oral and Maxillofacial Sciences, Sapienza University, Rone int

${ }^{4}$ Department of Sense Organs, Sapienza University, Rome, Italy

${ }^{5}$ Regulatory DPT Lofarma Milano, Milan, Italy

${ }^{6}$ Research DPT Lofarma Milano, Milan, Italy

\section{Corresponding author}

Franco Frati

Scientific and Medical DPT Lofarma Milano, Mila. It $\lrcorner$ ly

Phone: 00390258198212

E-mail: franco.frati@lofarma.it

\section{Summary}

Allergen immunotherapy (AIT, a aimed at inducing tolerance to allergens, such as pollens, dust mites or moulds, by administerivo 1 reasing amounts of the causative allergen through subcutaneous or sublingual route. The viderice of efficacy of AIT is high, but the issue of safety, especially for the subcutaneous route $\mathrm{ul}$ t be taken into account. The search for safer AIT products aimed at reducing the allergenicitv, $n$ thus adverse reactions, while maintaining the immunogenicity, that is essential for effecti $\sim$ - ss, gave rise to the introduction of allergoids, which were conceived to fulfill these require. 'e. 's. In the first allergoids glutaraldehyde or formaldehyde were used as cross-linking agent to polyn erize allergens, this resulting in high molecular weight molecules $(200,000$ to $20,000,000$ daltons) which were significantly less allergenic due to a decreased capacity to bridge IgE on its 
specific receptor, while maintaining the immunogenicity and thus the therapeutic efficacy. In recent years further agents, acting as adjuvants, such as L-tyrosine, monophosphoryl lipid A, alיminium hydroxide, were added to polymerized extracts. Moreover, a carbamylated monomeric in ovid was developed and, once adsorbed on calcium phosphate matrix, used by subcutaneous 1 , ite. At the same time, in virtue of its peculiarities, such allergoid revealed particularly suitao e for sublingual administration. A lot of clinical evidences show that it is well tolerated, ?arg ${ }^{2} \mathrm{v}$ safer and effective. Importantly, the higher safety of allergoids allows faster treatment sed tles that favor patient compliance and, according to pharmaco-economic studies, they mig ${ }_{1}+b=$ more cost-effective than other AIT options.

\section{Key words}

Allergen immunotherapy; safety; efficacy; allergoids; nor omeric allergoids

\section{Background}

Allergen immunotherapy (AIT) was intre du $d$ in 1911 by Noon and Freeman, with the provisional name of "desensitizing vaccine"[1]. This treatment was aimed at reducing the reactivity to allergens, namely grass pollen, by subcutane $\mathrm{s}$ s : dministration of increasing amounts of the causative allergen, but remained for decades merely smpirical. The discovery of IgE antibodies in the 1960s [2] was crucial for the developmen of s. ientific knowledge on the mechanism of allergy, leading to a marked improvement in the di gnc sis but also in the quality of allergen extracts for AIT [3). The introduction in the 1980s of iminun therapy products of high biological potency was a further step towards the quality impro ment and the consequent reliability of AIT, but the issue of safety came to light. Reports of $\mathrm{t}^{\prime-1}$ reactions to subcutaneous immunotherapy from the UK [4] and the USA [5] were publishe, Inducing to reappraise, especially in patients with allergic rhinitis, the feasibility of a treatment burdened by the risk of severe adverse reactions. Such an issue motivated the search for safer AIT products, intending to reduce the allergenicity, and thus adverse reactions while 
maintaining the immunogenicity that is essential to induce the immunological modification associated with effective AIT. The first approach to reach this goal was accomplished by introducing the allergoids, conceived to fulfill such the requirements, then followed by a dose red $x$ in in coadministration of the allergen dosage concomitant to adjuvants, and by routes ac ninistration different from the injective route.

\section{The evolution of allergoids for subcutaneous immunotherapy}

The first study on allergoids obtained by polymerization of allergens us. , ght taraldehyde as a crosslinking agent dates back to 1973 [6]. Such chemical treatment resu ${ }^{\circ}$ in high molecular weight molecules (200,000 to 20,000,000 daltons) which were significantly $\mathrm{L}$ s allergenic due to a decreased capacity to bridge IgE on its specific receptor while maint in $\mathrm{g}$ the immunogenicity and thus the therapeutic efficacy. After 10 years of studies, Grammer et a concluded that this approach was the most successful in providing a good balance of safety, effj zacy and, and immunogenicity in multiple clinical trials [7]. In Europe, the allergoids obta ea the treatment of the partially purified pollen extracts with formaldehyde were evaluated. i. 1932 Puttonen et al. showed that the formaldehyde treatment resulted in a change of the net ha. - of proteins to the more acidic site, in a considerable reduction of the activities of natur lly occurring enzymes of native allergen extracts, and the observation of only a trace of acti i y $n$ the RAST inhibition assay [8]. In the study by Bousquet et al. a lyophilized extract of grass + - 'len was dissolved in a phosphate buffer, adding formaldehyde to the solution to obtain a $10_{1 .}-1 / \mathbf{1}^{1}$ pollen extract. After incubation, the solution was dialyzed at +4 " C to remove formaldeh, de nd lyophilized. The product was administered by a rush schedule and compared to SCIT with a common standardized grass extract. Both treatments were effective on grass induced rhinit mure severe reactions were observed with the standardized extract, but also patients treated wi $\mathrm{n}$ is allergoid had SRs [9]. The reduction but not abolition of SRs was also confirmed with oth $r$ kinds of allergoids, such as the formalinized alum-absorbed allergoid. In a double-blind, placebo-controlled study on patients with grass-pollen allergy high doses of grass allergoid, corresponding to a cumulative pre-seasonal dosage of 46,050 protein nitrogen units (PNU), were 
administered, with only one systemic reaction. All patients were evaluated before and during the treatment by symptom-medication scores, specific nasal and skin reactivity, and immunological (specific IgE, IgG, IgG1 and IgG4 antibodies) parameters. The actively treated pa: eltis had significantly lower symptom-medication scores than placebo during the month of $\mathrm{N} \cdot \mathrm{v} \cdot \mathrm{a} \cdot \mathrm{d}$ showed a significant decrease in specific skin and nasal reactivity, and a significant early in s $r$ ase in specific $\operatorname{IgE}, \operatorname{IgG}, \operatorname{IgG} 1$, and $\operatorname{IgG} 4$, with a subsequent decrease of $\operatorname{IgE}$ and $\operatorname{IgG} 1\ulcorner 1 \mathrm{C}$, n similar aluminum hydroxide-adsorbed depot allergen preparation produced by allergen ni lific ation by formaldehyde and titrated in therapeutic units (TU) was studied in a placebo-contron r rial on children with grass pollen-induced allergic rhinitis. Children in the immunotherapy grou received 7 injections of grass pollen allergoid before grass pollen season and remained on maintenance treatment 27 months. Clinical and laboratory parameters were compared between the active and placebo-treated groups. After 1 year of immunotherapy, the rhino-conjunctivit sy nptom-medication score was significantly lower in the immunotherapy group, and skin tes rentity and nasal reactivity to grass pollen were significantly decreased. Grass-specific IgG, Ig ₹1 : nd IgG4 increased significantly already at the end of the s build-up therapy, while the seasc $\mathrm{a}_{1}$-..rease in IgE was blunted by active treatment [11]. A recent double-blind, placebo-control'ed t ial evaluated the dose-response relationship of the same allergoid preparation comparing a s n ı ı species (Phleum pratense) and a multiple species mixture. Three doses of $P$. pratense alle. roid (1800 TU, standard-dose $6000 \mathrm{TU}$ and $18000 \mathrm{TU})$ were compared with placebo anc the marketed 6-grass pollen allergoid (6000 TU). The primary endpoint was the change in wea size in response to the intra-cutaneous testing before and after treatment, while secondary outcomes wf ie the change in total nasal symptom score measured assessed in the allergen exposure char ar, the changes in P. pratense-specific IgG4 and the incidence of adverse events. All three dose, $\mathrm{C}_{1}$ the P. pratense and the 6-grass pollen allergoid preparations were significantly superior to p $^{1}$ ace $\mathrm{p}$ ior the primary endpoint, while no significant differences in the change in nasal scores were detected. The high-dose of $P$. pratense, when compared to the standard-dose, did not yield any additional significant benefit, but was associated with a slight increase in adverse reactions [12]. 
Further allergoid preparations include the addition to polymerization (by glutaraldehyde or formaldehyde) of L-tyrosine and monophosphoryl lipid A, aluminum hydroxide.

Henmar et al. performed a direct comparison of three intact allergen extracts and fo 1 liurgoids using IgE inhibition and basophil activation assays to measure the allergenicity, $\mathrm{H}_{\mathrm{n}} \mathrm{man} \mathrm{T}$ cell proliferation and specific IgG-titres following mouse immunizations to assess imın in Jgenicity of all products. The results showed important differences in both allergenicity anc inmnunogenicity, that

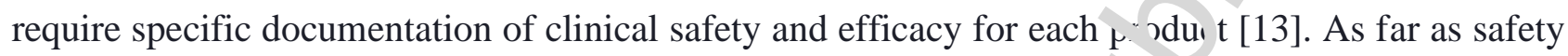
is concerned, the Paul-Ehrlich-Institute published a report on advere Irug reactions (ADRs) to injective immunotherapy from 1991 to 2000 . ADRs to allergoids clas tied as serious were evaluated between $0.01 \%$ and $0.0005 \%$, corresponding to one seriour $n$ h in 10,000 to 200,000 injections. "Although based only on absolute numbers, the hypothetica. assumption regarding better tolerance of the allergoids compared to native allergen prepa atic as was not confirmed, while concerning delayed ADRs $75 \%$ of them were related to $\mathrm{h}$ - mu $\mathrm{d}$ ied semi-depot preparations, and $25 \%$ were related to allergoids [14]. In a recent review by 'aja xulendran et al. on novel strategies for AIT, which analyzed the data from grass pollen allerson currently available, the pharmaco-economic aspects were also considered. Based on the a vail ble studies, the authors concluded that allergoids, mainly based on their shorter schedules a a aministration, might be more cost-effective than other AIT options [15].

\section{The development of allery ia for sublingual immunotherapy}

A particular allergoid o be administered by sublingual route has been developed. and used for almost 30 years. The produut $v$ sed was a carbamylated monomeric allergoid, which is a chemically modified allergen obtai 1 vy substitution of $\varepsilon$-aminogroups of allergen lysine residues, which reduces $\operatorname{IgE}$ binding ar iv 1.7 while preserving immunogenicity. Initially this allergoid was used for subcutaneous routa $[1 / 7$ unce adsorbed into a matrix of calcium phosphate; at the same time the peculiarities (monomericity) of this allergoid made it particularly suitable for sublingual administration. The definition of monomeric derives from the selectivity of carbamylation, which does not concern the 
structural conformation, with no increase of the size of the allergen molecule as occurs with polymerization. The first double-blind, placebo-controlled trial on the efficacy of an allergoid administered by the sublingual route was published into Lancet as a demonstration of $\mathrm{i}^{\dagger} \_$_ iginality. In patients with mite-induced rhinitis, active treatment resulting in significantly ow $r$ symptom scores and a significant decrease of the immune-mediated inflammatory response $[1 /]$. The second trial evaluated the efficacy of sublingual tablets of monomeric allergoid shta $\eta_{\epsilon}$ from grass pollen in children with rhinitis and asthma caused by grass pollen. Children $\mathrm{r}$ - eivi $\mathrm{ig}$ a preseasonal active treatment had a significant reduction of symptoms scores, particulan, $\mathrm{b}$-onchial symptoms, and a decrease of nasal eosinophil cationic protein, with good tolerance to : e allergoid [18]. The safety in children was confirmed in subjects aged less than 5 years 1 te teu with either mite of grass pollen monomeric allergoids [19]. A further safety study evaluatea 105 patients (28 children and 77 adults) undergoing SLIT with a mite or grass pollen or Parie 'ria pollen by an ultra-rush schedule reaching the top dose in 20 minutes. Only one patient ( $9 \%$, nad an adverse reaction consisting of gastric pyrosis, with spontaneous recovery [20]. Inde. ', ' everal other studies on the efficacy and safety of monomeric allergoids are available, whiř....e analyzed in 2010 by Mösges et al., in a systematic review and meta-analysis. The glob $1 \mathrm{n}$ n mber of patients with allergic rhinitis included in these studies were 266 for grass poller : nc 241 mite allergoid. The average improvement in symptom scores was $34 \%$ for grass pollen $\cdots$ d $22 \%$ for mite allergoid in comparison with the placebo group, and the average improven. $n t$ medication scores was $49 \%$ and $24 \%$ for grass pollen and mite allergoid, respectively $\mathrm{Fe} / \mathrm{side}$ effects, with no systemic reactions, were reported in the trials [21]. The most recent stulues investigated the dose-dependence and dose-finding of monomeric allergoids. The first stud - varuated the efficacy and safety of the dose of 1000 or 2000 allergy units (AU) in 34 mite allers $1 \mathbb{C}_{\mathrm{r}} \mathrm{t}$ 1ents, using as primary outcome the change of the threshold of allergen concentration indreing a positive nasal provocation test. After 12 weeks all patients treated with 1000 AU and all but one treated with 2000 AU had an increase in the threshold dose inducing positive provocation tests. The rate of adverse reactions, all mild, was comparable with the two doses [22]. In a 
randomized, double-blind, phase 2 study on 158 adult patients with grass pollen-induced rhinoconjunctivitis, four different doses, equal to 300, 600, 1000 and $2000 \mathrm{UA} / \mathrm{dav}$ were administered. The rate of patients with no symptoms to conjunctival provocation test af $\backsim$ r tratment was 54.3, 47.6, 59.0 and 51.4\%, respectively, suggesting 1000 UA/day as the optima ' dos. No serious adverse event was reported [23]. However, in a 12-week double-blind, placelc-crintrolled dosefinding study on 131 patients with mite-induced rhino-conjunctivitis recei in thu dose of 300,1000 , 2000. Or $3000 \mathrm{UA} /$ day, the highest rate of treatment response, as a ess $d$ by the conjunctival provocation test, was observed with the $2000 \mathrm{UA} /$ day $(88.5 \%)$. An ov ra I number of 20 treatmentrelated adverse events (all mild) were recorded [24]. The posı ve clinical outcomes of the carbamylated monomeric allergoid are supported by immun su rical investigations, which disclosed that the mechanisms of action are those illustrated for $A_{1}{ }^{T}$ in general. In fact, SLIT with mite monomeric allergoid was shown to down-regulate al ${ }^{2} \operatorname{rg} € \mathrm{n}$-specific $\operatorname{IgE}$ and to increase interferongamma- and interleukin (IL)-10 production, con n $\mathrm{n}_{--1}^{-1}$ associated with the development of allergen tolerance [25]. The up-regulation of IL-10 wa det scted also during a short-term course (60 days) of SLIT with grass monomeric allergoid, alrag with allergen-specific T-cell proliferation and reduction of allergen-specific in vitro proliferat on [?6]. In a study comparing two induction schedules of SLIT with mite monomeric allergoid of $\mathrm{d} f \mathrm{ff}$ :rent duration (98 days vs. 16 days) the more rapid induction scheme was associated with a rec vtion in TNF-alpha and IL-4 at the end of induction [27].

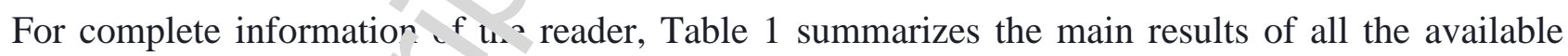
studies on SLIT with ( arbc mylated monomeric allergoid,

\section{Conclusions}

The introduct on allergoids was an actual advance for AIT with inhalant allergens, providing a response $\uparrow+1$ problem of systemic reactions to injective immunotherapy, which rather commonly hincerea the performance of the treatment, being rarely able even to result in fatal events. Abundant literature supports the role of allergoids in AIT, including for injective AIT several types, obtained by different chemical treatments of the natural allergens to reduce allergenicity while maintaining the 
immunogenicity and thus the therapeutic efficacy. Also, a product to be used by the sublingual route is available, which consists of the carbamylated monomeric allergoid, which has good evidence of efficacy and safety. Still, there is room for allergoids characterization, taking into an ount the allergoids require more sophisticated analytical methods than native extracts [28]. \& aa' 'ition, in the current landscape of the regulatory requests governing allergen products, special $r$ : $\mathrm{a}$ irements need to be implemented for control of allergoids [29]. We have identified a tota oi 24 journal articles reporting 313 participants as total number of active patients and $298 \mathrm{pa}$. icip ants as total number of placebo/control group (Lais Mites: 64 active/ 61 placebo-control ; Laı. B rch 55 active /82 placebocontrol; Lais Grass 114 active/ 95 placebo-control; Lais Parietaria 8u ctive/ 60 placebo-control).

\section{ETHICS APPROVAL AND CONSENT TO \& ^ R' 'ICIPATE}

Not applicable.

\section{CONFLICT OF INTEREST}

$\mathrm{CC}$, MS declare no conflict of int $\lrcorner \mathrm{r}$ st, financial or otherwise. C Incorvaia is a scientific consultant for Stallergenes Italy. FF, CE ( ${ }_{-}$MG are employees of Lofarma SPA.

\section{CRediT AUTHOR STATI:LN:}

IC, CC, CE, FF, MG Con eptualization, Resources. CC, IC Writing - Original Draft. FF, GA, MS Writing - Review \& Ed ting

FUNDING A CKIVUWLEDGEMENTS: declared none. 


\begin{tabular}{|c|c|c|c|c|c|c|c|}
\hline Allergen & Study & $\begin{array}{l}\text { Study } \\
\text { objective }\end{array}$ & Study design & \begin{tabular}{|l|} 
Scheme \\
-Duration- \\
Dose \\
\end{tabular} & No patient & Patology & Results \\
\hline \multirow{4}{*}{$\begin{array}{l}\text { Lais Mites - } \\
\text { Chemically } \\
\text { Modified } \\
\text { Allergen } \\
\text { Extract of } \\
\text { house dust } \\
\text { mites } \\
\text { (Dermatopha } \\
\text { goides } \\
\text { pteronyssinu } \\
\text { s 50\%, } \\
\text { Dermatopha } \\
\text { goides } \\
\text { farinae 50\%) }\end{array}$} & $\begin{array}{l}\text { Pacor ML } \\
\text { (1995) [30] }\end{array}$ & $\begin{array}{l}\text { Efficacy } \\
\text { and safety }\end{array}$ & $\begin{array}{l}\text { Open observational } \\
\text { Study }\end{array}$ & \begin{tabular}{|l|} 
Continuative - \\
2 years- increasing \\
doses $25 / 100 / 300 / 1000$ \\
AU alternate days, \\
each dose for 3 times; \\
maintenance dose: 1 \\
tablet of $1000 \mathrm{AU}$ \\
weekly.
\end{tabular} & $14 /-$ & $\begin{array}{l}\text { Asthma } \\
\text { of light or } \\
\text { moderate } \\
\text { degree }\end{array}$ & 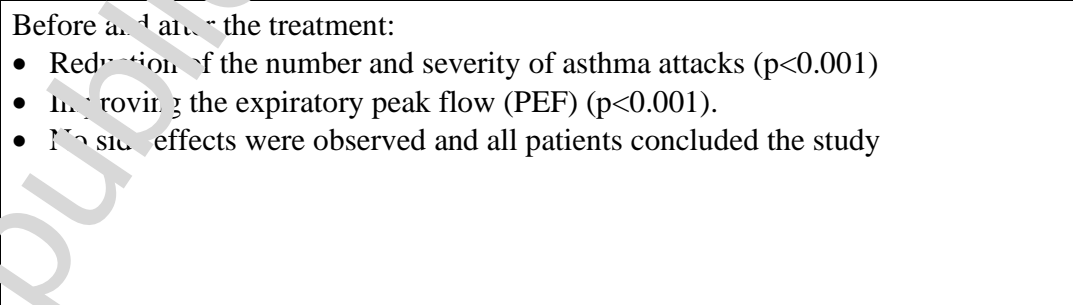 \\
\hline & $\begin{array}{l}\text { Passalacqua } \\
\text { G(1998) } \\
{[17]}\end{array}$ & $\begin{array}{l}\text { Efficacy } \\
\text { and safety }\end{array}$ & $\begin{array}{l}\text { Randomised, } \\
\text { placebo controlled, } \\
\text { double-blind, } \\
\text { parallel study }\end{array}$ & $\begin{array}{l}\text { Continuative - } 24 \\
\text { months- increasing } \\
\text { doses } 25 / 50 / \\
100 / 200 / 300 / 600 / 1000 \\
\text { AU alternate days, } \\
\text { each dose for } 3 \text { times; } \\
\text { maintenance dose: } 2 \\
\text { tablets of } 1000 \text { AU } \\
\text { twice weekly. }\end{array}$ & $\begin{array}{l}10 \text { Active / } \\
9 \text { Placebo }\end{array}$ & $\begin{array}{l}\text { Pe nic } \\
\text { rhinoce iuncti } \\
\text { ritis, at } \\
\text { lea for } 2 \\
\text { rear. }\end{array}$ & $\begin{array}{l}\text { Active vs Placebo: } \\
\text { - Neutrophilic infiltration decreased }(\mathrm{p}=0 \cdot 002) \text {. } \\
\text { - Eosinophilic infiltration decreased before challenge }(\mathrm{p}=0 \cdot 001) \text {. } \\
\text { - ICAM- } 1 \text { expression reduced before challenge }(\mathrm{p}=0 \cdot 01) \text { and during and after } \\
\text { treatment }(\mathrm{p}=0 \cdot 002) \\
\text { - ECP decreased after } 12 \text { months of treatment }(\mathrm{p}=0 \cdot 04) \\
\text { - The treatment was well tolerated. } 1 \text { local (oral itching) side-effects in active } \\
\text { group }\end{array}$ \\
\hline & $\begin{array}{l}\text { Lombardi } \\
(2001)[31]\end{array}$ & Safety & $\begin{array}{l}\text { Observational } \\
\text { Study }\end{array}$ & $\begin{array}{l}\text { Continuative } \\
-31.9 \text { months - } \\
\text { increasing doses } 25 / \\
50 / \\
100 / 200 / 300 / 600 / 16 \text {, } \\
\text { for } 8 \text { weeks eve y } \\
\text { other day; maint, nan } \\
\text { dose } 2000 \text { A } J \text { re a } \\
\text { week. }\end{array}$ & & $\begin{array}{l}\text { Perennial or } \\
\text { seasonal } \\
\text { rhinitis and/or } \\
\text { mild asthma }\end{array}$ & $\begin{array}{l}\text { - } 17 \text { adverse events corresponding to } 7.5 \% \text { of patients and } 0.52 \text { per } 1000 \text { doses: } 7 \\
\text { episodes of rhinitis, } 3 \text { of oral itching, and } 1 \text { of abdominal pain. Two cases of } \\
\text { urticaria and two of abdominal pain/nause were controlled by a temporary dose- } \\
\text { adjustment, and one case of urticaria and conjunctivitis required oral antihistamines. } \\
\text { - Medical intervention was needed in six patients only during a 3-year period. } \\
\text { - No severe systemic side-effect } \\
\text { *The events reported as results of Lombardi's study were observed in } 198 \text { patients } \\
\text { receiving different SLIT treatments (69 patients - Mites ; } 75 \text { patients - Grasses; } 46 \text { - } \\
\text { Parietaria; } 4 \text { Birch; } 1 \text { Olive; } 3 \text { Compositae) }\end{array}$ \\
\hline & $\begin{array}{l}\text { Passalacqua } \\
\text { G(2006) } \\
{[32]}\end{array}$ & $\begin{array}{l}\text { Efficacy } \\
\text { and } \\
\text { Safety }\end{array}$ & $\begin{array}{l}\text { Randomized, } \\
\text { placebo-controlled, } \\
\text { double-blind, } \\
\text { multicenter }\end{array}$ & $\begin{array}{l}\text { Continuat } \\
-2 \text { ear } \\
\text { Inci sin, doses } 25 / \\
\text { no/200/300/600/1000 } \\
\text { AL on alternate days, } \\
\text { e. ch dose for } 3 \text { times; } \\
\text { maintenance dose: } 1 \\
\text { tablet of } 1000 \text { AU } \\
\text { twice weekly. }\end{array}$ & $34 / 34$ & $\begin{array}{l}\text { Mild persistent } \\
\text { rhinitis } \\
\text { with/without } \\
\text { mild } \\
\text { intermittent } \\
\text { asthma, } \\
\text { since at least } 2 \\
\text { years }\end{array}$ & $\begin{array}{l}\text { Active vs Placebo: } \\
\text { - Fifty-six patients completed the study (28 Active/ } 28 \text { Placebo) } \\
\text { - A significant difference in the clinical score after } 1 \text { year of treatment }(\mathrm{P}=0.027) \\
\text { - A significant difference for the symptom nasal obstruction after } 1 \text { year }(\mathrm{P}=0.05) \\
\text { and } 2 \text { years }(\mathrm{P}=0.033) \\
\text { - A significant global drug intake at the first year of treatment }(\mathrm{P}=0.036) \\
\text { - A significant change in SLIT group was seen for the item change in health status } \\
(\mathrm{P}=0.05) \text { after the second year of treatment. } \\
\text { - No relevant side effect was reported ( } 30 \text { vs } 43 \text { events) } \\
\text { - The need for extra visits was lower in the active group }(25 \% \text { vs } 43 \%)\end{array}$ \\
\hline
\end{tabular}




\begin{tabular}{|c|c|c|c|c|c|c|c|}
\hline Allergen & Study & $\begin{array}{l}\text { Study } \\
\text { objective }\end{array}$ & Study design & \begin{tabular}{|l|} 
Scheme \\
-Duration- \\
Dose \\
\end{tabular} & No patient & Patology & Results \\
\hline & \begin{tabular}{|l} 
Cosmi L \\
$(2006)[25]$
\end{tabular} & Efficacy & $\begin{array}{l}\text { Open, randomized, } \\
\text { two arm parallel } \\
\text { group: one treated } \\
\text { with SLIT, } \\
\text { one untreated } \\
\text { (UT) and } \\
\text { receiving only } \\
\text { rescue } \\
\text { symptomatic } \\
\text { drugs }\end{array}$ & $\begin{array}{l}\text { Continuative } \\
-1 \text { year and half - } \\
\text { increasing doses }(25 / \\
50 / \\
100 / 200 / 300 / 600 / 1000 \\
\text { AU for } 8 \text { weeks every } \\
\text { other day; } \\
\text { the maintenance dose } \\
\text { of } 1000 \text { AU once a } \\
\text { week. }\end{array}$ & $\begin{array}{l}12 \text { SLIT- } \\
\text { treated/ } 13 \\
\text { untreated } \\
\text { (UT) }\end{array}$ & $\begin{array}{l}\text { Perennial } \\
\text { rhinitis } \\
\text { and/or rhinitis } \\
\text { plus mild } \\
\text { asthma }\end{array}$ & 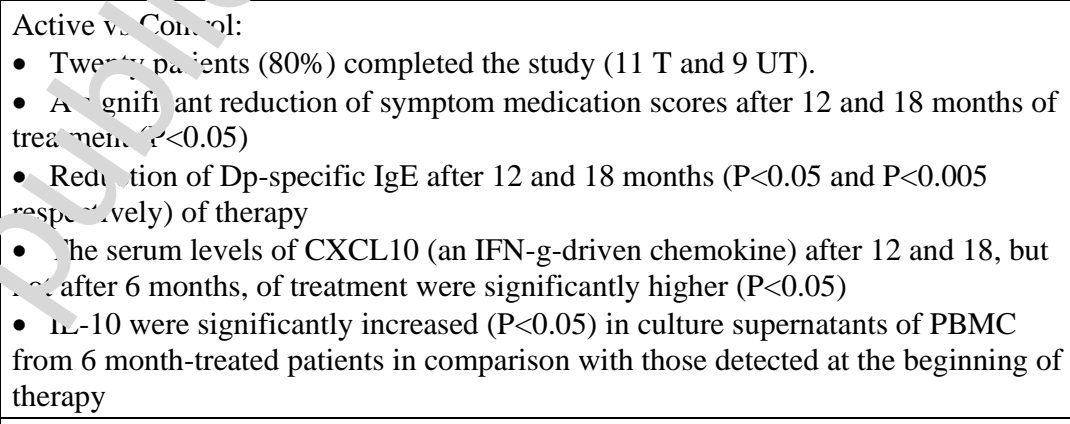 \\
\hline & $\begin{array}{l}\text { Giordano T } \\
\text { (2006) [33] }\end{array}$ & $\begin{array}{l}\text { Efficacy } \\
\text { and safety }\end{array}$ & $\begin{array}{l}\text { Open observational } \\
\text { study }\end{array}$ & $\begin{array}{l}\text { Continuative }-1 \text { year- } \\
\text { Four-day build-up }: 1^{\text {st }} \\
\text { day } 500 \mathrm{AU}, 2^{\text {nd }} \text { day } \\
1.000 \mathrm{AU}, 3^{\text {rd }} \text { day } \\
1500 \mathrm{AU}, 4^{\text {th }} \text { day } 2000 \\
\text { AU . Maintenance: } 5- \\
365 \text { day } 1000 \mathrm{AU} \\
\text { twice weekly }\end{array}$ & 27 & $\begin{array}{l}\text { mo rac' } \\
\text { severe 'initis, } \\
\text { vith or not } \\
\text { mu'rate } \\
\text { sth na, } \\
\text { nerennial or } \\
\text { easonal }\end{array}$ & $\begin{array}{l}\text { - Improvement of the VAS scores was observed. } \\
\text { - Decrease of the drug consumption }\{\mathrm{p}<0.01) \text {. } \\
\text { - No side effects: Only two mild adverse reactions: somnolence and tiredness } \\
\text { *The study observed } 39 \text { patients house-dust mite (n. } 27) \text {, grass pollen (n. 7), olive } \\
\text { pollen (n. 3), cat dander (n. 1) and Parietaria pollen (n. 1). }\end{array}$ \\
\hline & $\begin{array}{l}\text { D'Anneo RW } \\
(2010)[34]\end{array}$ & $\begin{array}{l}\text { Efficacy } \\
\text { and } \\
\text { Safety }\end{array}$ & $\begin{array}{l}\text { Prospective, open- } \\
\text { label, randomized } \\
\text { study included two } \\
\text { parallel groups one } \\
\text { treated with SLIT, } \\
\text { one treated with } \\
\text { standard pharmaco } \\
\text {-therapy (control } \\
\text { group) }\end{array}$ & $\begin{array}{l}\text { Continuative } \\
-12 \text { months - } \\
300 \text { AU tablet each } \\
\text { day for } 4 \text { day and the } \\
\text { 12-month; } \\
\text { maintenance dose } 2,10 \\
\text { AU/week }\end{array}$ & & \begin{tabular}{l|} 
Intermittent or \\
persistent \\
rhinitis or rhino \\
conjunctivitis \\
and/or \\
intermittent, \\
mild-persistent \\
or persistent \\
moderate- \\
severity \\
allergic asthma
\end{tabular} & $\begin{array}{l}\text { SLIT group vs Control: } \\
\text { - All patients very well tolerated both the four-day build-up phase and the } 12- \\
\text { month maintenance phase } \\
\text { - Visual Analogue Scale rises significantly, about } 45 \% \text {, in both groups }(\mathrm{p}=0.001) \text {. } \\
\text { - Reduction in the global symptom score SLIT group vs control group, about } 52 \% \\
\text { ( } \mathrm{p}=0.0004) \text {. } \\
\text { - Smaller rescue drug consumption SLIT group vs control group, about } 9 \% \text {. } \\
\text { The difference between before SLIT (T0) and after } 12 \text { months }(\mathrm{Tl}) \text { was highly } \\
\text { significant in skin reactivity ( } \mathrm{p}=0.000003) \text {. The control group had a small increase } \\
\text { in skin-reactivity }(2.6 \pm 15.7 \%) \text { with significance between } \mathrm{T} 0 \text { and } \mathrm{Tl}(\mathrm{p}=0.5226) \text {. }\end{array}$ \\
\hline $\begin{array}{l}\text { Lais } \\
\text { Betulle- } \\
\text { Chemically } \\
\text { modified }\end{array}$ & $\begin{array}{l}\text { Burastero SE } \\
(2009)[35]\end{array}$ & $\begin{array}{l}\text { Efficacy } \\
\text { and } \\
\text { Safety }\end{array}$ & $\begin{array}{l}\text { Open } \\
\text { observational, } \\
\text { parallel } \\
\text { grouped: active } \\
\text { and placebo }\end{array}$ & $\begin{array}{l}\text { Continua ve } \\
-6 \text { r tonus - } \\
1.0 w \text { AI every day }\end{array}$ & $11 / 11$ & $\begin{array}{l}\text { Seasonal } \\
\text { allergic rhino } \\
\text { conjunctivitis } \\
\text { with or not } \\
\text { mild asthma }\end{array}$ & $\begin{array}{l}\text { - Two patients had transient itching in their mouth, spontaneously disappeared. } \\
\text { - During the pollen season symptoms/drug usage scores improved of } 30 \% \text { and } \\
40 \% \text { respectively in actively treated and control patients }(\mathrm{p}<0.0001) \text {; well-days } \\
\text { (days without intake of rescue medications and symptoms score less than } 2 \text { ) were in } \\
33 \% \text { and } 23 \% \text { of patients respectively }(\mathrm{p}=0.0024) \text {. }\end{array}$ \\
\hline
\end{tabular}




\begin{tabular}{|c|c|c|c|c|c|c|c|}
\hline Allergen & Study & $\begin{array}{l}\text { Study } \\
\text { objective }\end{array}$ & Study design & $\begin{array}{l}\text { Scheme } \\
\text {-Duration- } \\
\text { Dose }\end{array}$ & No patient & Patology & Results \\
\hline \multirow[t]{3}{*}{$\begin{array}{l}\text { allergen } \\
\text { extract of } \\
\text { trees } \\
\text { pollens } \\
\text { (Betula } \\
\text { pendula } \\
50 \% \text {, } \\
\text { Alnus } \\
\text { incana } \\
50 \% \text { ) }\end{array}$} & $\begin{array}{l}\text { L. } \\
\text { Bommarito } \\
(2009)[36]\end{array}$ & Efficacy & $\begin{array}{l}\text { Open, randomized, } \\
\text { parallel group: } \\
\text { three active } \\
\text { groups }\end{array}$ & $\begin{array}{l}\text { Continuative } \\
-18 \text { months- } \\
\text { pre-coseasonal } \\
\text { regimen (T1) }(6,000 \\
\text { AU/week for } 10 \\
\text { weeks/year for two } \\
\text { years); perennial } \\
\text { regimen (T2) (2,000 } \\
\text { AU/week for the entire } \\
\text { study period) } \\
\end{array}$ & $\begin{array}{l}8 \mathrm{~T} 1+8 \mathrm{~T} 2 \\
15 \mathrm{~T} 3 \\
\text { (Drug } \\
\text { Therapy } \\
\text { alone) }\end{array}$ & $\begin{array}{l}\text { Allergic } \\
\text { rhinoconjuncti } \\
\text { vitis } \\
\text { with/without } \\
\text { mild } \\
\text { intermittent } \\
\text { asthma }\end{array}$ & 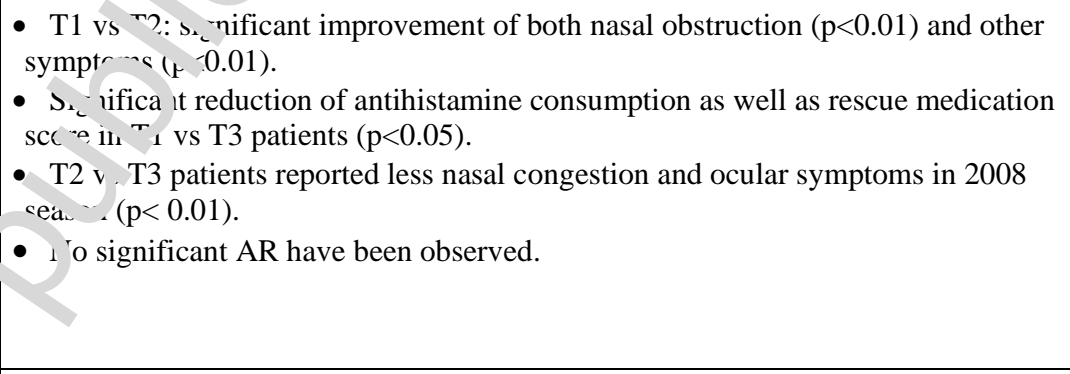 \\
\hline & \begin{tabular}{|l} 
Passali D \\
$(2010)$ [37]
\end{tabular} & $\begin{array}{l}\text { Efficacy } \\
\text { and } \\
\text { Safety }\end{array}$ & \begin{tabular}{|l|} 
Prospective, \\
open, randomized \\
study, with three \\
parallel groups and \\
control group
\end{tabular} & $\begin{array}{l}\text { Continuative } \\
-6 \text { months - } \\
\text { 1,000 AU (Group A); } \\
500 / 1,000 / 1500 \mathrm{AU} \\
\text { up-dosing in 4-day } \\
\text { (Group B); } \\
\text { 300/600/900/1200 AU } \\
\text { (Group C) up-dosing } \\
\text { in 4-day; } \\
\text { Maintenance: } 1,000 \\
\text { AU 5-7 times a week }\end{array}$ & $\begin{array}{l}4 \text { (Group } \\
\text { A) / } 3 \\
\text { (Group B) / } \\
3 \text { (Group } \\
\text { C) / } 3 \\
\text { (control) }\end{array}$ & $\begin{array}{l}\text { Rhiniti anc } \\
\text { oc lo-1 initi }\end{array}$ & $\begin{array}{l}\text { Treated VS Control } \\
\text { - All patients tolerated all the three dosage very well, no patient interrupted } \\
\text { - A statistically significant }(\mathrm{p}<0.02) \text { reduction of SMSs vs control group } \\
\text { - Significant }(\mathrm{p}<0.01) \text { decrease in nasal reactivity the three SLI T-treated groups, } \\
\text { while the untreated controls remained unchanged } \\
\text { - A significant increase in VAS values has been observed in all } 3 \text { study groups, in } \\
\text { comparison to the controls }(\mathrm{p}<0.001) \text {. } \\
\text { - During up-dosing } 4 \text { slight side-effects in } 4 \text { patients, } 1 \text { somnolence and } 1 \\
\text { tiredness, and } 2 \text { oral itching. No side-effects were recorded during the maintenance } \\
\text { treatment. }\end{array}$ \\
\hline & $\begin{array}{l}\text { Marogna M } \\
\text { (2013) [38] }\end{array}$ & $\begin{array}{l}\text { Efficacy } \\
\text { and } \\
\text { Safety }\end{array}$ & $\begin{array}{l}\text { Open } \\
\text { randomized } \\
\text { parallel } 4 \\
\text { groups study: } \\
\text { Group 1: BUD 400 } \\
\text { mcg/day + anti Lt/s } \\
\text { Group 2: BUD } 800 \\
\text { mcg/day } \\
\text { Group 3: BUD } \\
1600 \text { mcg/day } \\
\text { Group } 4: \text { BUD } \\
400 \text { mcg/day + } \\
\text { SLIT }\end{array}$ & $\begin{array}{l}\text { Discontinuos - } 3 \\
\text { seasons of treatment } \\
\text { (February to April) } \\
\text { four-day } \\
\text { build-up phase } \\
\text { followed by a } \\
\text { maintenance pr se of } \\
\text { three years, } 100 \\
\text { Allergic Unit unce a } \\
\text { day fo ve } \\
\text { days' ak }\end{array}$ & $\begin{array}{l}\text { Jup } \\
\text { u= 1)/ } \\
\text { uoup } 2 \\
\mathrm{n}=21) / \\
\text { Group } 3 \\
(\mathrm{n}=21) / \\
\text { Group } 4 \\
(\mathrm{n}=21)\end{array}$ & \begin{tabular}{l|} 
Seasonal mild \\
and persistent \\
asthma and \\
normal lung \\
function \\
associated with \\
AR
\end{tabular} & $\begin{array}{l}\text { - A significantly performance associated with the use of SLIT; only patients of } \\
\text { group 4, achieved an appreciable control (mean } 24 ; \text { SEM 0.242). } \\
\text { - A significant improvement in allergy symptoms-medications scores (SMS), } \\
\text { in patients of group } 4 \text { (decrease of } 87 \% \text { ) than in all other groups (p < } 0.01) \text {. } \\
\text { - The FEV1 increase and the albuterol intake in group } 4 \text { was significantly lower } \\
\text { after three years ( }<0.001) \text {, } \\
\text { - Reduction of nasal eosinophils and nasal corticosteroids in group } 4 \\
\text { - Significant difference in the PD20 was detected at baseline between the controls } \\
\text { and the } 1,000 \text { AU and between the } 1,000 \text { and } 2,000 \text { AU groups } \\
\text { - During the three years of SLIT course, two patients reported one episode of } \\
\text { occurred during the maintenance phase and self-resolved without any therapy in less } \\
\text { than two hours. }\end{array}$ \\
\hline $\begin{array}{l}\text { Lais } \\
\text { Grasses- } \\
\text { Chemically } \\
\text { modified } \\
\text { allergen }\end{array}$ & $\begin{array}{l}\text { Bordignon V } \\
\text { (1994) [39] }\end{array}$ & Efficacy & $\begin{array}{l}\text { Randomised, } \\
\text { placebo-controller, } \\
\text { double-blind } \\
\text { parallel study }\end{array}$ & $\begin{array}{l}\text { iscoutinuos }-3 \\
\text { sea ons of treatment } \\
\text { (f ebruary to April) - } \\
25 / 100 / 300 \text { and } 1,000 \\
\text { AU every other day (3 } \\
\text { times a week) }\end{array}$ & $30 / 30$ & \begin{tabular}{|l|} 
Perennial rhino \\
conjunctivitis \\
and/or asthma \\
at least for 2 \\
years
\end{tabular} & $\begin{array}{l}\text { Active vs Placebo: } \\
\text { - A statistically significant reduction of nasal and bronchial symptoms particularly } \\
\text { after the second and the third years of treatments }(\mathrm{p}<0.01) \text {. } \\
\text { - Significant reduction of drugs consumption }(\mathrm{p}<0.01)\end{array}$ \\
\hline
\end{tabular}




\begin{tabular}{|c|c|c|c|c|c|c|c|}
\hline Allergen & Study & $\begin{array}{l}\text { Study } \\
\text { objective }\end{array}$ & Study design & \begin{tabular}{|l|} 
Scheme \\
-Duration- \\
Dose \\
\end{tabular} & No patient & Patology & Results \\
\hline \multirow{4}{*}{$\begin{array}{l}\text { extract of } \\
\text { grass } \\
\text { pollens } \\
\text { (Holcus } \\
\text { lanatus } \\
\text { 33\%, } \\
\text { Phleum } \\
\text { pratense } \\
\text { 33\%, Poa } \\
\text { pratensis } \\
33 \% \text { ) }\end{array}$} & $\begin{array}{l}\text { Pacor M.L. } \\
\text { (1996) [40] }\end{array}$ & Efficacy & $\begin{array}{l}\text { Open non } \\
\text { comparative }\end{array}$ & \begin{tabular}{|l|} 
Discontinuos -6 pre- \\
seasonal months for 2 \\
years- increasing doses \\
$25 / 100 / 300$ up to 1,000 \\
AU every other day ( 3 \\
times a week) \\
\end{tabular} & 34 & $\begin{array}{l}\text { Seasonal rhino } \\
\text { conjunctivitis }\end{array}$ & 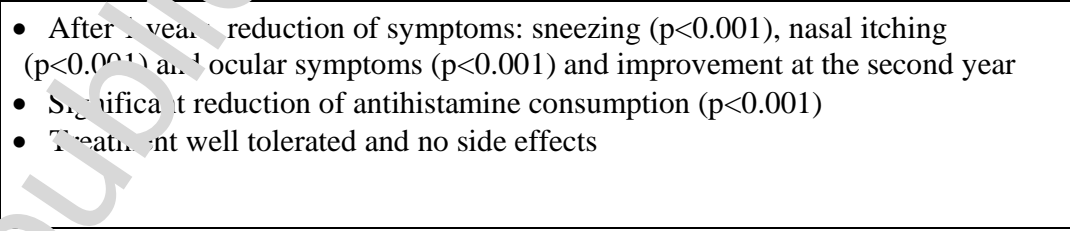 \\
\hline & $\begin{array}{l}\text { Caffarelli } C . \\
(2000) \text { [18] }\end{array}$ & $\begin{array}{l}\text { Efficacy } \\
\text { and safety }\end{array}$ & $\begin{array}{l}\text { Randomised, } \\
\text { double-blind, } \\
\text { placebo-controlled } \\
\text { study }\end{array}$ & \begin{tabular}{l|} 
Continuative- \\
3 months before pollen \\
season- increasing \\
doses \\
$25 / 50 / 100 / 200 / 300 / 600$ \\
and $1,000 \mathrm{AU}$ \\
every other day ( 3 \\
times a week)
\end{tabular} & $\begin{array}{l}24 \text { active / } \\
24 \text { placebo }\end{array}$ & \begin{tabular}{l|} 
Seasonal \\
rhinitis and/ or \\
rhino- \\
conjunctivit. \\
and/or \\
br nrh. \\
asthit.
\end{tabular} & $\begin{array}{l}\text { Act ve vs Placebo: } \\
44 \text { out of } 48 \text { patients }(91.6 \%) \text {, all } 24 \text { in the active treatment group and } 20 \text { of } 24 \\
\text { given placebo, completed the study: three because they moved away, and one } \\
\text { because of a mild side-effect (abdominal pain) } \\
\text { - Significant reduction of total symptoms }(\mathrm{P}<0.05) \text { during the pollen season } \\
\text { - Treatment well tolerated and compliance was good } \\
\text { - EG2/EGl increased significantly only in the placebo group during natural } \\
\text { allergen exposure }(\mathrm{P}<0.01)\end{array}$ \\
\hline & $\begin{array}{l}\text { Lombardi } C \\
(2001)[41]\end{array}$ & $\begin{array}{l}\text { Efficacy } \\
\text { and safety }\end{array}$ & $\begin{array}{l}\text { Open, controlled } \\
\text { study }\end{array}$ & $\begin{array}{l}\text { Discontinuos }-3 \\
\text { months of } \\
\text { pre-seasonal } \\
\text { treatment for } \\
3 \text { years (1995- } \\
\text { 1997) - cumulative } \\
\text { dosage, } \\
\text { 36,000 AU }\end{array}$ & $\begin{array}{l}26 \\
\text { (pharmacn- } \\
\text { therapy }- \\
\text { SLI' } \\
25 \\
\left(p^{\prime} \text { armi. }: 0-\right. \\
\text { the. nv } \\
\left.0^{\prime}, y^{y}\right)\end{array}$ & $\begin{array}{l}\text { Seas nal } \\
\text { itis } \\
\text { und/or asthma } \\
\text { (mild } \\
\text { intermittent or } \\
\text { mild persistent) }\end{array}$ & $\begin{array}{l}\text { Active vs Control: } \\
\text { - Significant increase }(\mathrm{p}=.0 .01) \text { of } \mathrm{PD} 20 \text { at the methacholine } \\
\text { - Significant clinical improvement both for rhinitis }(\mathrm{p}=0.001) \text { and asthma } \\
(\mathrm{p}=0.001) \\
\text { - Reduction of drug intake }(\mathrm{p}=0.001) \\
\text { - Improvement of rhinitis symptom without modification of drug intake } \\
\text { - Treatment well tolerated and no relevant side effects during the } 3 \text { years. }\end{array}$ \\
\hline & $\begin{array}{l}\text { Lombardi C } \\
(2001)[31]\end{array}$ & Safety & $\begin{array}{l}\text { Observational } \\
\text { Study }\end{array}$ & 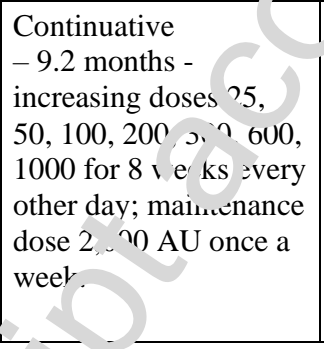 & $5 /-$ & $\begin{array}{l}\text { Perennial or } \\
\text { seasonal } \\
\text { rhinitis and/or } \\
\text { mild asthma }\end{array}$ & $\begin{array}{l}\text { - } 17 \text { adverse events corresponding to } 7.5 \% \text { of patients and } 0.52 \text { per } 1000 \text { doses: } 7 \\
\text { episodes of rhinitis, } 3 \text { of oral itching, and } 1 \text { of abdominal pain. Two cases of } \\
\text { urticaria and two of abdominal pain/nause were controlled by a temporary dose- } \\
\text { adjustment, and one case of urticaria and conjunctivitis required oral antihistamines. } \\
\text { - Medical intervention was needed in six patients only during a 3-year period. } \\
\text { - No severe systemic side-effect } \\
\text { *The events reported as results of Lombardi's study were observed in } 198 \text { patients } \\
\text { receiving different SLIT treatments (69 patients - Mites ; } 75 \text { patients - Grasses; } 46 \text { - } \\
\text { Parietaria; } 4 \text { Birch; } 1 \text { Olive; } 3 \text { Compositae) }\end{array}$ \\
\hline
\end{tabular}




\begin{tabular}{|c|c|c|c|c|c|c|c|}
\hline Allergen & Study & $\begin{array}{l}\text { Study } \\
\text { objective }\end{array}$ & Study design & \begin{tabular}{|l|} 
Scheme \\
-Duration- \\
Dose \\
\end{tabular} & No patient & Patology & \begin{tabular}{|l} 
Results \\
\end{tabular} \\
\hline & $\begin{array}{l}\text { Quercia O } \\
\text { (2001) [42] }\end{array}$ & $\begin{array}{l}\text { Efficacy } \\
\text { and safety }\end{array}$ & $\begin{array}{l}\text { Prospective, } \\
\text { randomized, } \\
\text { open controlled } \\
\text { trial with three } \\
\text { parallel groups. }\end{array}$ & $\begin{array}{l}\text { Continuative for } 16 \\
\text { days: } 25 / 100 / 300 / 1000 \\
\text { AU. } \\
\text { After for 2 years: } \\
\text { Continuative Group } 1 \\
1,000 \text { AU/week - } \\
\text { Pre-seasonal Group 2: } \\
\text { 5,000 AU/week for } 10 \\
\text { weeks/year, on demand } \\
\text { drug therapy alone } \\
\text { (Group 3) for 2 years } \\
\end{array}$ & $\begin{array}{l}\text { Group } 1 \\
(\mathrm{n}=10) \\
\text { Group } 2 \\
(\mathrm{n}=11) \\
\text { and Group } \\
3(\mathrm{n}=11)\end{array}$ & $\begin{array}{l}\text { Rhino- } \\
\text { conjunctivitis } \\
\text { with/without } \\
\text { mild } \\
\text { intermittent } \\
\text { asthma }\end{array}$ & 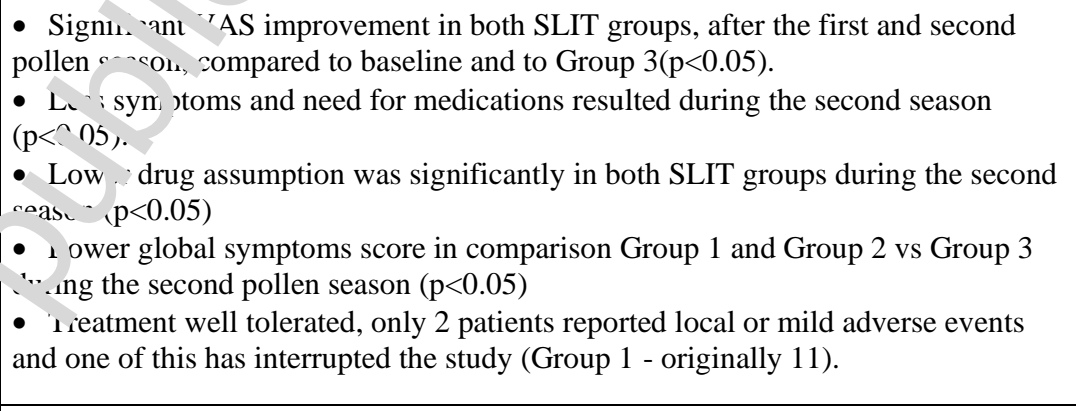 \\
\hline & $\begin{array}{l}\text { A.G. Palma } \\
\text { Carlos } \\
(2006) \text { [43] }\end{array}$ & $\begin{array}{l}\text { Efficacy } \\
\text { and safety }\end{array}$ & $\begin{array}{l}\text { Monocentric } \\
\text { randomised, } \\
\text { double-blind, } \\
\text { placebo controlled }\end{array}$ & $\begin{array}{l}\text { Discontinuos - pre- } \\
\text { seasonal months for } 2 \\
\text { years }-25,100,300 \\
\text { and } 1,000 \text { AU every } \\
\text { other day ( } 3 \text { times a } \\
\text { week) for } 14 \text { weeks } \\
1,000 \text { AU } 2 \text { times a } \\
\text { week till May. }\end{array}$ & $\begin{array}{l}17 \text { Active / } \\
16 \text { Placebo }\end{array}$ & $\begin{array}{l}\text { Se snn } 1 \\
\text { rhiru njuncti } \\
\text { vitis with or } \\
\text { inter nittentor } \\
\text { nund persistent } \\
\text { sthmas since } \\
\text { at least two } \\
\text { years }\end{array}$ & $\begin{array}{l}\text { Active vs Placebo: } \\
\text { - } 20 \text { patients out of the } 33 \text { enrolled }(60.6 \% \text { ) completed the study (13 Active/ } 7 \\
\text { Placebo) } \\
\text { - Statistically significant decrease of symptom scores (conjunctivitis } \mathrm{p}<0.02 \text {, } \\
\text { rhinorrea }<<0.03 \text { and sneezing } \mathrm{p}<0.03 \text { ) } \\
\text { - Statistically significant decrease of nasal reactivity at the second year of } \\
\text { treatment ( } \mathrm{p}<0.03 \text { ) } \\
\text { - Lower consumption of inhaled steroids, mean monthly scores ( } \mathrm{P}<0.02 \text { ) } \\
\text { - Treatment well tolerated; } 2 \text { mild local adverse events occurred without } \\
\text { interruption of therapy }\end{array}$ \\
\hline & $\begin{array}{l}\text { Burastero, } \\
\text { S.E }(2008) \\
{[26]}\end{array}$ & Efficacy & $\begin{array}{l}\text { Open, } \\
\text { observational pilot } \\
\text { study }\end{array}$ & $\begin{array}{l}\text { Continuative } \\
-60 \text { days - dose of } \\
2,000 \text { AU once a da }\end{array}$ & & $\begin{array}{l}\text { Rhinoconjuncti } \\
\text { vitis with or } \\
\text { not mild } \\
\text { asthma for at } \\
\text { least } 2 \text { years }\end{array}$ & $\begin{array}{l}\text { - Decrease in Allergen-Specific Proliferation to the } \mathrm{rPhl} \mathrm{p} 1 \text { and to the raw grass } \\
\text { extract after } 2 \text { Months of SLIT }(\mathrm{P}=.002 \text { and } .04) \\
\text { - Increase in Transcription of IL-10 }(\mathrm{P}<.001) \text { and TGF- } \beta(\mathrm{P}=.06) \text {, at } \mathrm{rPhl} \mathrm{p} 1- \\
\text { Stimulated Lymphocytes } \\
\text { - Correlation indexes of pre-treatment and post-treatment changes in IL-10 vs } \\
\text { TGF- } \beta \text { expression were } 0.17(\mathrm{P} .47) \text { and } 0.16(\mathrm{P} .70) \text {, respective }\end{array}$ \\
\hline $\begin{array}{l}\text { Lais } \\
\text { Parietaria- } \\
\text { Chemically } \\
\text { modified } \\
\text { allergen } \\
\text { extract of } \\
\text { parietaria } \\
\text { pollens }\end{array}$ & \begin{tabular}{|l} 
Ariano $R$ \\
(1998) [44]
\end{tabular} & $\begin{array}{l}\text { Efficacy } \\
\text { and safety }\end{array}$ & $\begin{array}{l}\text { Randomised, } \\
\text { placebo controlled, } \\
\text { double-blind } \\
\text { parallel study. }\end{array}$ & 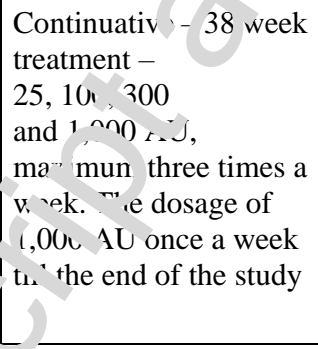 & $15 / 15$ & $\begin{array}{l}\text { Allergic } \\
\text { rhinitis } \\
\text { with or without } \\
\text { asthma }\end{array}$ & $\begin{array}{l}\text { Active vs Placebo: } \\
\text { - Improvement of score symptoms and drug consumption with a statistically } \\
\text { significant difference at the end of the treatment }(\mathrm{p}<0.01) \\
\text { - Comparison of the areas of the skin tests and RAST before and after treatment } \\
\text { showed no statistically significant difference in the two groups. } \\
\text { - Comparison of nasal or bronchial provocation test before and after treatment with } \\
\text { statistically significant difference ( }<0.05 \text { ) } \\
\text { No side effect observed: one patient of active group discontinued the treatment } \\
\text { owing to digestive troubles (Active Group - } 14 \text { out of } 15 \text { completed the study) }\end{array}$ \\
\hline
\end{tabular}




\begin{tabular}{|c|c|c|c|c|c|c|c|}
\hline Allergen & Study & $\begin{array}{l}\text { Study } \\
\text { objective }\end{array}$ & Study design & \begin{tabular}{|l|} 
Scheme \\
-Duration- \\
Dose \\
\end{tabular} & No patient & Patology & Results \\
\hline \multirow[t]{5}{*}{$\begin{array}{l}\text { (Parietaria } \\
\text { judaica } \\
\mathbf{5 0 \%} \\
\text { Parietaria } \\
\text { officinalis } \\
\mathbf{5 0 \%} \text { ) }\end{array}$} & $\begin{array}{l}\text { Lombardi C } \\
\text { (2001) [31] }\end{array}$ & Safety & $\begin{array}{l}\text { Observational } \\
\text { Study }\end{array}$ & \begin{tabular}{l|} 
Continuative \\
-16.3 months - \\
increasing doses 25, \\
$50,100,200,300,600$ \\
1000 for 8 weeks every \\
other day; maintenance \\
dose 2,000 AU once a \\
week.
\end{tabular} & $46 /-$ & $\begin{array}{l}\text { Perennial or } \\
\text { seasonal } \\
\text { rhinitis and/or } \\
\text { mild asthma }\end{array}$ & 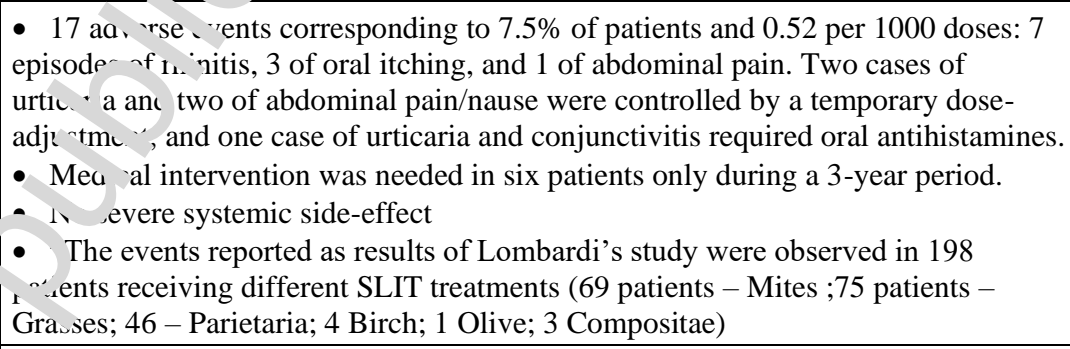 \\
\hline & $\begin{array}{l}\text { Arena A } \\
\text { (2003) [45] }\end{array}$ & $\begin{array}{l}\text { Efficacy } \\
\text { and } \\
\text { tolerability }\end{array}$ & $\begin{array}{l}\text { Prospectic } \\
\text { Observational } \\
\text { Study }\end{array}$ & $\begin{array}{l}\text { Continuative - } \\
\text { Three Years - } \\
\text { increasing doses } \\
25 / 50 / 100 / 300 \text { AU and } \\
1000 \text { AU for } 3 \\
\text { alternate days. } \\
\text { Maintenance phase } \\
\text { most patients received } \\
2000 \text { AU twice weekly } \\
\end{array}$ & $\begin{array}{l}24 \text { SLIT / } \\
11 \text { SIT / } 9 \\
\text { pharmacolo } \\
\text { gical } \\
\text { therapy }\end{array}$ & $\begin{array}{l}\text { Rhinitis and/e. } \\
\text { mild } \\
\text { int nit nt or } \\
\text { persisu nt } \\
\text { asthma or } \\
\text { cos. 'nnctivitis }\end{array}$ & $\begin{array}{l}\text { - } 8 \text { patients interrupted the immunotherapy during the study period: } 3 \text { SLIT group } \\
\text { and } 5 \text { SIT group } \\
\text { - The physician's opinion on efficacy, by symptoms and drug consumption } \\
\text { reduction, was statistically better in the SLIT group than in the other two groups ( } \mathrm{p}< \\
0.0001 \text { ). } \\
\text { - The difference between the patient's degree of satisfaction of treatments was } \\
\text { statistically significant in favour of SLIT treatments ( } \mathrm{p}<0.0001 \text { ). } \\
\text { * The events reported as results of a study observed in } 110 \text { patients receiving } \\
\text { different treatments (Parietaria, Graminacea, Olea, Dermathopaghoides) }\end{array}$ \\
\hline & $\begin{array}{l}\text { Lombardi C } \\
\text { (2004) [46] }\end{array}$ & Safety & $\begin{array}{l}\text { Multicenter } \\
\text { observational } \\
\text { Study }\end{array}$ & $\begin{array}{l}\text { Continuative }-18 \pm 2 \\
\text { weeks- } 1000 \mathrm{AU} \\
\text { tablets - count: } \\
3952 / 4050 \text { tablet }\end{array}$ & & $\begin{array}{l}\text { Allergic } \\
\text { rhinitis and/or } \\
\text { asthma at least } \\
2 \text { years }\end{array}$ & $\begin{array}{l}\text { - } 11 \text { mild side effects were reported in } 6(7 \% *) \text { patients: } 6 \text { oral itching, } 2 \text { rhinitis, } 2 \\
\text { nausea, and } 1 \text { generalized itching } \\
\text { - Omitted dose was documented in } 11 \text { patients. } \\
\text { *on a total of } 86 \text { patients: } 41 \text { received SLIT to mite and } 45 \text { to pollens ( } 24 \text { grasses, } 18 \\
\text { Parietaria, } 3 \text { Ragweed). }\end{array}$ \\
\hline & $\begin{array}{l}\text { Gammeri E } \\
\text { (2005) [20] }\end{array}$ & $\begin{array}{l}\text { Safety and } \\
\text { the } \\
\text { tolerability }\end{array}$ & $\begin{array}{l}\text { Open sequential } \\
\text { Non controlled }\end{array}$ & 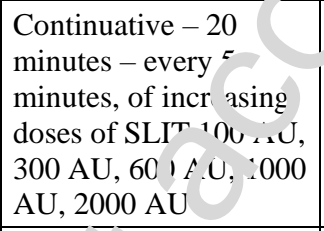 & 4 & \begin{tabular}{l|} 
intermittent/per \\
sistent rhinitis \\
or intermittent/ \\
mild persistent \\
asthma
\end{tabular} & $\begin{array}{l}\text { Only } 1 \text { patient out of } 105 *(0.9 \%) \text { had a mild local symptom (gastric pyrosis) that } \\
\text { occurred } 30 \text { minutes after the last initial dose and spontaneously disappeared as the } \\
\text { treatment was continued. } \\
\text { *The study observed } 105 \text { patients [Dust }(n=56) \text {, Parietaria }(n=34) \text { and Timothy- } \\
\text { grass }(n=15) \text { ] }\end{array}$ \\
\hline & $\begin{array}{l}\text { La Grutta S } \\
\text { (2007) [47] }\end{array}$ & Efficacy & $\begin{array}{l}\text { Prospective, open- } \\
\text { controlled } \\
\text { randomised }\end{array}$ & 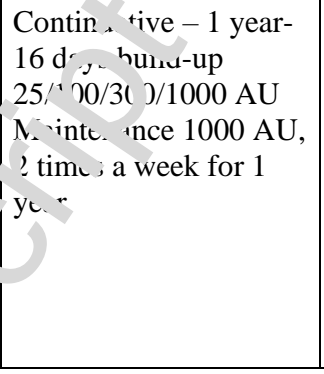 & $\begin{array}{l}33 \text { SLIT / } \\
23 \text { Control } \\
\\
* 56 \mathrm{pt} \\
\text { allergic to } \\
\text { House Dust } \\
\text { mite with } \\
\text { (n-36) or } \\
\text { without } \\
\text { Parietaria } \\
\end{array}$ & $\begin{array}{l}\text { mild persistent } \\
\text { asthma with or } \\
\text { not moderate } \\
\text { intermittent } \\
\text { moderate } \\
\text { rhinitis }\end{array}$ & $\begin{array}{l}\text { Active vs Control } \\
\text { - All patients completed the study } \\
\text { - Greater reduction daily of the mean symptom score }(\mathrm{p}<0.01) \text { and drug } \\
\text { consumption }(\mathrm{p}<0.001) \text { in the SLIT than in the control group. } \\
\text { - MCh PD20 increased only in the SLIT group }(\mathrm{p}<0.0005) \\
\text { - The reduction of nasal eosinophils was statistically greater }(\mathrm{P}<0.05) \text { only in the } \\
\text { SLIT group. }\end{array}$ \\
\hline
\end{tabular}




\begin{tabular}{|c|c|c|c|c|c|c|c|}
\hline Allergen & Study & $\begin{array}{l}\text { Study } \\
\text { objective }\end{array}$ & Study design & $\begin{array}{l}\text { Scheme } \\
\text {-Duration- } \\
\text { Dose }\end{array}$ & No patient & Patology & Results \\
\hline & $\begin{array}{l}\text { D'Anneo } R W \\
\text { (2008) [48] }\end{array}$ & $\begin{array}{l}\text { Efficacy } \\
\text { and safety }\end{array}$ & $\begin{array}{l}\text { Prospective, } \\
\text { randomized, } \\
\text { With three parallel } \\
\text { Groups receiving } \\
\text { either two different } \\
\text { dosages of SLIT or } \\
\text { the standard } \\
\text { chronic }\end{array}$ & $\begin{array}{l}\text { Continuative }-6 \\
\text { months - } \\
1,000 \mathrm{AU} / \text { week - } \\
3,000 \mathrm{AU} / \text { week }\end{array}$ & $\begin{array}{l}24 \text { (SLIT } \\
1,000 \\
\text { AU/week) / } \\
21 \text { (SLIT } \\
3,000 \\
\text { AU/week) / } \\
21 \text { (drug } \\
\text { therapy) }\end{array}$ & \begin{tabular}{|l|} 
Seasonal \\
rhinoconjuncti \\
vitis and/or \\
asthma \\
(mild \\
intermittent or \\
mild persistent)
\end{tabular} & 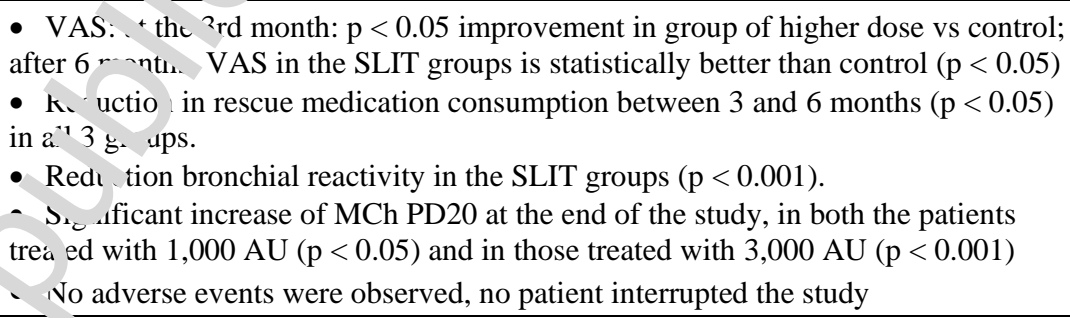 \\
\hline & $\begin{array}{l}\text { Passali D } \\
\text { (2010) [37] }\end{array}$ & $\begin{array}{l}\text { Safety and } \\
\text { efficacy }\end{array}$ & $\begin{array}{l}\text { Prospective, } \\
\text { open, randomized } \\
\text { study, with three } \\
\text { parallel groups and } \\
\text { control group }\end{array}$ & $\begin{array}{l}\text { Continuative }-6 \\
\text { months - 1,000 AU } \\
\text { (Group A) - 4-day up- } \\
\text { dosing 500/1000/1500 } \\
\text { AU (Group B) - 4-day } \\
\text { up-dosing 300/600/900 } \\
\text { /1200 AU (Group C) } \\
\text { Maintenance: } 1,000 \\
\text { AU 5-7 times a week }\end{array}$ & $\begin{array}{l}4 \text { (Group } \\
\text { A) /3 } \\
\text { (Group B) / } \\
2 \text { (Group } \\
\text { C) / } 2 \\
\text { (Control) }\end{array}$ & $\begin{array}{l}\text { Rhinitis ar } \\
\text { oculo-rh:-.itis }\end{array}$ & $\begin{array}{l}\text { Treated VS Control } \\
\text { - All patients tolerated all the three dosage very well, no patient interrupted } \\
\text { - A statistically significant }(\mathrm{p}<0.02) \text { reduction of SMSs vs control group } \\
\text { - Significant }(\mathrm{p}<0.01) \text { decrease in nasal reactivity the three SLI T-treated groups, } \\
\text { while the untreated controls remained unchanged } \\
\text { - A significant increase in VAS values has been observed in all } 3 \text { study groups, in } \\
\text { comparison to the controls }(\mathrm{p}<0.001) \text {. } \\
\text { - During up-dosing } 4 \text { slight side-effects in } 4 \text { patients, } 1 \text { somnolence and } 1 \\
\text { tiredness, and } 2 \text { oral itching. No side-effects were recorded during the maintenance } \\
\text { treatment. }\end{array}$ \\
\hline
\end{tabular}




\section{References}

1) Frew AJ. Hundred years of allergen immunotherapy. Clin Exp Allergy. 2011; 41(9): 1'21 1225.

2) Johansson SG, Bennich $\mathrm{H}$, Wide L. A new class of immunoglobulin in hi mas serum. Immunology. 1968;14(2):265-72.

3) Berings M, Karaaslan C, Altunbulakli C, Gevaert P, Akdis M, Bachert C et a1. Advances and highlights in allergen immunotherapy: On the way to sustained clinical and immunologic tolerance. J Allergy Clin Immunol. 2017;140(5): ? ? 1267.

4) Committee on Safety in Medicine. CSM update: desensitizing ac ines. BMJ 1986; 293:948.

5) Lockey RF, Benedict LM, Turkeltaub PC, Bukantz SC. Fat tlities trom immunotherapy (IT) and skin testing (ST). J Allergy Clin Immunol 1987;79:6 10- 7.

6) Patterson R, Suszko IM, McIntire FC. Polymeli ed ragweed antigen E. I. Preparation and immunologic studies. J Immunol 1973;110(5):1 †2-12.

7) Grammer LC, Shaughnessy MA, Patters (n I . Modified forms of allergen immunotherapy. J Allergy Clin Immunol. 1985;76(2 Pt 2) 277401

8) Puttonen E, Maasch HJ, Pilström I. N wa.dies of allergen and allergoid preparations from purified

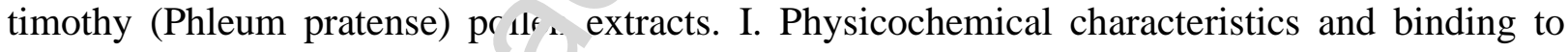
allergen-specific human Ig- Int Arch Allergy Appl Immunol. 1982;68(1):1-6.

9) Bousquet J, Hejjaoui A sal sa-Brociek W, Guérin B, Maasch HJ, Dhivert H, et al. Double-blind, placebo-controlled innnı.notherapy with mixed grass-pollen allergoids. I. Rush immunotherapy with allergoid: .11 1 standardized orchard grass-pollen extract. J Allergy Clin Immunol. $1987 ; 80(4): 5>1-" ;$.

10) Pastore"1' LA, Pravettoni V, Incorvaia C, Mambretti M, Franck E, Wahl R, et al. Clinical and ims u nogical effects of immunotherapy with alum-absorbed grass allergoid in grass-pollenindu ed hay fever. Allergy. 1992;47(4 Pt 1):281-90. 
11) Keskin O, Tuncer A, Adalioglu G, Sekerel BE, Saçkesen C, Kalayci O. The effects of grass pollen allergoid immunotherapy on clinical and immunological parameters in children with allergic rhinitis. Pediatr Allergy Immunol. 2006;17(6):396-407.

12) Pfaar O, Hohlfeld JM, Al-Kadah B, Hauswald B, Homey B, Hunzelmann N, et c Lu e-response relationship of a new Timothy grass pollen allergoid in comparison $w$ th a 6-grass pollen allergoid. Clin Exp Allergy. 2017;47(11):1445-1455..

13) Henmar H, Lund G, Lund L, Petersen A, Würtzen PA. Allergenicit, imr lunogenicity and doserelationship of three intact allergen vaccines and four allergoid va $\cdot$ cives for subcutaneous grass pollen immunotherapy. Clin Exp Immunol. 2008;153(3):316-23.

14) Lüderitz-Püchel U, Keller-Stanislawski B, Haustein D. ^vo buwertung des Risikos von:709-18 Test- und Therapieallergenen. Eine Analyse der U.W Meldungen von 1991 bis 2000. Bundesgesundheitsbl Gesundheitsforsch Gesundh itss shutz 2001;44.

15) Rajakulendran M, Tham EH, Soh JY, Varı 3e.. HP. Novel strategies in immunotherapy for allergic diseases. Asia Pac Allergy. 2018;0 (?): 14.

16) Galimberti M., Cantone R., Pastore M., M.ıstrello G. e Falagiani P."Immunotherapy with grass allergoid (Modall). Preliminary r sult ". Italian Journal of Chest Diseases, Suppl. 6 (Nov / Dec. 1986).

17) Passalacqua G, Albano M I gonese L, Riccio A, Pronzato C, Mela GS, et al. Randomised controlled trial of lo sa' a argoid immunotherapy on allergic inflammation in mite-induced rhinoconjunctiviti: La lcet. 1998 28;351(9103):629-32.

18) Caffarelli C, Sensi LG, Marcucci F, Cavagni G. Preseasonal local allergoid immunotherapy to grass pol'. 111 children: a double-blind, placebo-controlled, randomized trial. Allergy. $\left.2000 ; 55_{1} ?\right): 1142-7$

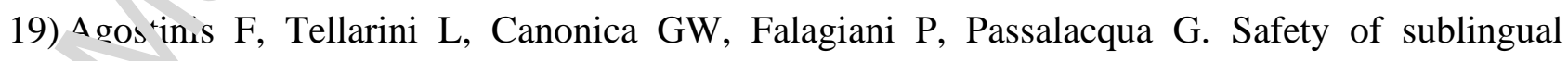
immunotherapy with a monomeric allergoid in very young children. Allergy. 2005;60(1):133 
20) Gammeri E, Arena A, D'Anneo R, La Grutta S. Safety and tolerability of ultra-rush (20 minutes) sublingual immunotherapy in patients with allergic rhinitis and/or asthma. Allergol Immunopathol (Madr). 2005 Jul-Aug;33(4):221-3.

21) Mösges R, Ritter B, Kayoko G, Allekotte S. Carbamylated monomeric allergoic as a therapeutic option for sublingual immunotherapy of dust mite- and grass pollen- in luced allergic rhinoconjunctivitis: a systematic review of published trials with a mem analysis of treatment using Lais ${ }^{\circledR}$ tablets. Acta Dermatovenerol Alp Pannonica Adriat. $2 \iota^{1}$ 0;1! (3):3-10.

22) Scalone G, Compalati E, Bruno ME, Mistrello G. Effect of two av es of carbamylated allergoid extract of dust mite on nasal reactivity. Eur Ann Allergy Clin Im. ınol. 2013;45(6):193-200.

23) Mösges R, Rohdenburg C, Eichel A, Zadoyan G, Kasric Eı, Shah-Hosseini K, et al. Dosefinding study of carbamylated monomeric allergoid tabi ts in grass-allergic rhinoconjunctivitis patients. Immunotherapy. 2017;9(15):1225-1238.

24) Hüser C, Dieterich P, Singh J, Shah-Hosse i l, Allekotte S, Lehmacher W, et al. A 12-week DBPC dose-finding study with sublingual .'on meric allergoid tablets in house dust mite-allergic patients. Allergy. 2017;72(1):77-84

25) Cosmi L, Santarlasci V, Ange 1 R Liotta F, Maggi L, Frosali F, et al. E. Sublingual immunotherapy with Dermatc $p^{\prime}$ a , oides monomeric allergoid down-regulates allergen-specific immunoglobulin E and jncru $r e s$ both interferon-gamma- and interleukin-10-production. Clin Exp Allergy. 2006;36,$\cdot 2 u^{\prime}-72$

26) Burastero SE, Mi trel o G, Falagiani P, Paolucci C, Breda D, Roncarolo D, et al. Effect of sublingual immun therapy with grass monomeric allergoid on allergen-specific T-cell proliferati . an anterleukin 10 production. Ann Allergy Asthma Immunol. 2008;100(4):343-50.

27) Di Gi'arc'nino M, Perrone A, Petrarca C, Di Claudio F, Mistrello G, Falagiani P, et al. Early vton inc modulation after the rapid induction phase of sublingual immunotherapy with mite monomeric allergoids. Int J Immunopathol Pharmacol. 2008;21(4):969-76. 
28) Carnes J, Gallego MT, Moya R, Iraola V. Allergoids for allergy treatment. Recent Pat Inflamm Allergy Drug Discov. 2018;12(2):110-119.

29) Zimmer J, Bonertz A, Vieths S. Quality requirements for allergen extracts and sil. ovids for allergen immunotherapy. Allergol Immunopathol (Madr). 2017;45 Suppl 1:4

30) Pacor ML, Biasi D, Carletto A, Lunardi C. Effectiveness of oral immunothe ar y in bronchial asthma caused by Dermatophagoides pteronyssinus. Recenti Prog Mer 1;0), 66(12):489-491.

31) Lombardi C, Gargioni S, Melchiorre A, Tiri A, Falagiani P, Can nic: GW, et al. Safety of sublingual immunotherapy with monomeric allergoid in adults $\mathrm{r}$.ulticenter post-marketing surveillance study. Allergy 2001; 56:989-992.

32) Passalacqua G, Pasquali M, Ariano R, Lombardi C, Gi „u. 1 A, Baiardini I, et al. Randomized double blind controlled study with sublingual carbamy: ted allergoid immunotherapy in mild rhinitis due to mites. Allergy 2006; 61: 849-854.

33) Giordano T, Quarta C, Bruno ME, Falagi. ii : Riva G. Safety, tolerability and efficacy of sublingual allergoid immunotherapy with à 1-d y shortened build-up phase. Eur Ann Allergy Clin Immunol 2006; 38:310-2

34) D'Anneo RW, Bruno ME, Fala sian P. Sublingual allergoid immunotherapy: a new 4-day induction phase in patients al. gi - to house dust mites. Int J Immunopathol Pharmacol 2010; 23:553 -560.

35) Burastero S, Mistrello F, 'Tolucci C, Breda D, Roncarolo D, Zanotta S et al. Clinical and immunological co rela es of pre-co-seasonal sublingual immunotherapy with birch monomeric allergoid in patınts with allergic rhinoconjunctivitis. Int J Immunopathol Pharmacol. 2009; 22: 343-352.

36) Bomm arı L, Bruno ME, Nebiolo F, Moschella A, Zanierato G, Mistrello G, et al. Efficacy and cafet, of sublingual immunotherapy with birch monomeric allergoid: a comparison of two different treatment regimens versus pharmacological one. Allergy 2009;64(90) 99-178. 
37) Passali D, Mösges R, Passali GC, Passali FM, Ayoko G, Bellussi L. Safety, tolerability and efficacy of sublingual allergoid immunotherapy with three different shortened un-dosing administration schedules. Acta Otorhinolaryngol Ital. 2010;30(3):131-7.

38) Marogna M, Braidi C, Bruno ME, Colombo C, Colombo F, Massolo A, et al. Ti co tribution of sublingual immunotherapy to the achievement of control in birch-related mila 1 ier sistent asthma: A real-life randomised trial. Allergol Immunopathol (Madr). 2013;41(^):2: ^-¿4.

39) Bordignon V, Di Berardino L. Efficacy of a new oral immunoth apy for grass. Three years parallel study. Giorn. It. Allergol. Immunol. Clin. 1994; 4:153-15;

40) Pacor ML, Biasi D, Carletto A, Maleknia T, Lunardi C. Oral Imı. notherapy in the treatment of rhinoconjunctivitis due to grass pollen. Recenti Prog Med. 1 רu; 87:4-6.

41) Lombardi C, Gargioni S, Venturi S, Zoccali P, Canonica $\_W$, Passalacqua G. Controlled study of preseasonal immunotherapy with grass poller estract in tablets: Effect on bronchial hyperreactivity. J Invest Allergol Clin Immu $12051 ; 11(1): 41-45$.

42) Quercia O, Bruno ME, Compalati E, Falas: ’ni P, Mistrello G, Stefanini GF. Efficacy and safety of sublingual immunotherapy with gr..s .... onomeric allergoid: comparison between two different treatment regimens. Eur Ann Alle cgy Clin Immunol. 2011;43(6):176-83.

43) Palma Carlos AG, Santos AS, F ra ico-Ferreira M, Pregal AL, Palma Carlos ML, et al. Clinical efficacy and safety of prese ${ }^{\circ}$ rnal sublingual immunotherapy with grass pollen carbamylated allergoid in rhinitic rat ${ }^{\circ}$. A doubleblind placebo-controlled study. Allergol Immunopathol (Madr) 2006; 34:1 44-178.

44) Ariano R, Pan'an1 P.C, Augeri G. Efficacy and safety of oral immunotherapy in respiratory allergy to Pariet in judaica pollen. A double-blind study $\mathrm{J}$ Investig Allergol Clin Immunol. $1998 ;\{(3) .155-60$.

45) Aren. F, Barbatano E, Gammeri E, Bruno M, Riva G. Specific immunotherapy of allergic diseases: a three years perspective observational study. Int J Immunopathol Pharmacol 2003; $16: 277-82$ 
46) Lombardi C, Gani F, Landi M, Falagiani P, Bruno M, Canonica GW, et al. Quantitative assessment of the adherence to sublingual immunotherapy. J Allergy Clin Immunnl 2004; $113: 1219-20$

47) La Grutta S, Arena A, D’Anneo WR, Gamberi E, Leopardi S, Trimarchi A, et ، L raluation of the anti-inflammatory and clinical effects of sublingual immunotherapy $w$ th carbamylated allergoid in allergic asthma with or without rhinitis. A 12-month pu-suctive randomized, controlled, trial. Eur Ann Allergy Clin Immunol 2007; 39:40-4

48) D’Anneo RW, Arena A, Gammeri E, Bruno ME, Falagiani P, Riva G et al. Parietaria sublingual allergoid immunotherapy with a co-seasonal treatment schedule. 1 lergol Immunopathol (Madr). 2008 Mar-Apr;36(2):79-84 